\title{
Timing Recovery in Digital Subscriber Loops
}

\author{
OSCAR AGAZZI, MEMBER, IEEE, CHIN-PYNG JEREMY TZENG, DAVID G. MESSERSCHMITT, FELLOW, IEEE, AND \\ DAVID A. HODGES, FELLOW, IEEE
}

\begin{abstract}
Tradeoffs in the design of the timing recovery functions in a subscriber loop receiver are analyzed. The techniques considered are applicable to both the echo cancellation (EC) and time compression multiplexing (TCM) methods of full duplex transmission. Emphasis is on those techniques that lend themselves to implementation in MOSLSI technology, where the objective requirement is that timing recovery be implemented on a sampled-data signal (with the minimum possible sampling rate where $\mathrm{EC}$ is used).

The wave difference method (WDM) for timing recovery appears to be the best candidate. A detailed study of its performance is carried out analytically and by computer simulation for the case of binary and alternate mark-inversion (AMI) line coding. A closed form expression describing the binary jitter performance of the WDM and its continuous time counterpart, the spectral line technique, is used to compare the two techniques. Analytical and simulation results for recovered phase and jitter are presented for various cable pulse responses carefully chosen to represent worst-case or nearly worst-case conditions.
\end{abstract}

Two methods for including frequency detection in the WDM, the quadricorrelator and the rotational detector, are also simulated.

\section{INTRODUCTION}

$\mathbf{T}$ IMING recovery is one of the most critical functions that must be implemented in a digital subscriber loop receiver. Although there has been a lot of work on timing recovery [4] [16], this application poses new problems that deserve further analysis. Bridged taps, which are not found in the $T$-carrier systems that motivated much of the earlier work, affect both the recovered phase and the jitter of the timing signal. The desire to implement the transmitter and receiver in VLSI technology calls for sampled-data signal processing. If the echo canceler (EC) method [1] is used, timing must be derived from the received signal after echo cancellation,' and the sampling rate must be as low as possible in order to limit the complexity of the echo canceler. Similarly, in the time compression multiplexing (TCM) method the signal is also sampled at the frontend filters and equalizers, which would most likely be implemented in switched-capacitor techniques. In both cases, it is possible to approximate the continuous time case by increasing the sampling rate. In the EC approach this could be done by an interpolation filter located after the echo canceler, but this introduces extra complexity.

The subscriber loop application differs from voiceband data transmission in that both the central office and the subscriber transmitter are slaved to the central office clock. The fact that transmission is synchronous in the two directions also favors a

Paper approved by the Editor for Subscriber Loops and Services of the IEEE Communications Society for publication after presentation at GLOBECOM '84, Atlanta, GA, December 1984. Manuscript received December 22, 1983. This work was supported by grants from Advanced Micro Devices, Fairchild Semiconductor, Harris Corporation, National Semiconductor, and Racal-Vadic, with a matching grant from the University of California's MICRO program, and by a grant from Harris Semiconductor to CENICE.

O. Agazzi is with the Research and Development Service of the Argentine Navy (SENID) and the Argentine National Center for Electronic Component Research (CENICE), 1104 Buenos Aires, Argentina.

C.-P. J. Tzeng, D. G. Messerschmitt, and D. A. Hodges are with the Department of Electrical Engineering and Computer Sciences and the Electronics Research Laboratory, University of California, Berkeley, CA 94720. sampled-data timing recovery scheme. In voiceband data transmission, on the other hand, the two directions are asynchronous, so that it is desirable to reconstruct a continuous time waveform and resample it synchronously with the far-end clock, and timing recovery can be performed on the continuous time waveform.

This paper focuses on timing recovery in discrete time, and where the goal is to minimize the sampling rate. Also considered is sampled-data frequency detection in addition to phase detection, to increase the pull-in range of the phase-locked loop and allow the use of low-accuracy voltage-controlled oscillators (VCO's). Only binary and alternate mark-inversion (AMI) line coding are considered in this paper. Further work is in progress to assess the possible advantages of some form of partial-response line coding, as will be reported in a future paper.

Several timing recovery techniques have been analyzed in the literature. They can be classified into continuous-time and discrete-time techniques. Some of the most common continuous-time techniques are the spectral line [4], [5], [11], the threshold crossing [9], the sampled-derivative [9], the earlylate gate [6], [9], and the maximum likelihood estimation [9] techniques. In the spectral line technique, after a nonlinear operation on the data signal, a discrete line at the data rate is generated in the signal spectrum. This frequency component is separated from the residual continuous spectral components by using a narrow bandpass filter. The nonlinear operations most commonly used are squaring and full-wave rectification. Among the sampled data techniques, special consideration is given here to the wave difference method (WDM) [3] (similar to the early-late gate) and baud-rate sampling [7] techniques. No detailed analysis of performance of the WDM has been published, and some questions arise that deserve further study, as follows.

1) What is the timing phase recovered by this technique in the presence of severe pulse distortion, as can result, for example, from bridged taps? The answer to this question also depends upon the type of equalization used, as some methods are more sensitive to timing phase than others.

2) What is the jitter performance of the WDM?

3) What are the tradeoffs in the design of a phase-locked loop based on a WDM phase detector?

4) How can a frequency detector be designed in the context of the WDM, increasing the pull-in range of the PLL and decreasing the required free-running frequency accuracy of the VCO?

In Section II, phase and frequency detectors which use the WDM are characterized analytically. In Section III, the performance of the timing recovery system when operating with imperfections such as bridged taps is evaluated by computer simulation. In Section IV tradeoffs in the design of a PLL using a WDM phase/frequency detector are evaluated, including a design example.

\section{Analysis of the Wave Difference Timing RECOVERY TECHNIQUE}

In this section, the WDM is analyzed, extended to include a frequency detector, and shown under certain conditions to be the sampled-data equivalent of the spectral line method. 


\section{A. The Wave Difference Method}

Let

$$
s(t)=\sum_{k=-\infty}^{\infty} x_{k} h(t-k T)
$$

be the received signal in a baseband subscriber loop receiver where $h(t)$ is the channel response to the input pulse. In subsequent analytical results, binary line coding will be assumed, in which $x_{k}$ is an independent, identically distributed sequence of transmitted data symbols assuming the values -1 and +1 . In many of the simulation results, the additional case of AMI line coding will be considered. In this case $x_{k}$ can be considered to be the result of applying a first difference operation to an independent, identically distributed binary sequence assuming the values 0 and +1 .

Define the timing function as

$$
E\left\{f\left[\sum_{k=-\infty}^{\infty} x_{k} h(t-k T)\right]\right\}=w(t)
$$

where $f(\cdot)$ is some convenient nonlinear function, and $E$ stands for expected value. The timing function $w(t)$ is clearly periodic with period $T$, and so its spectrum consists of a set of discrete lines at multiples of the data rate. For the particular case $f(x)=x^{2}$,

$$
w(t)=E\left\{\left[\sum_{k} x_{k} h(t-k T)\right]^{2}\right\}=\sum_{k=-\infty}^{\infty} h^{2}(t-k T)
$$

where we have assumed that the $x_{k}$ are independent and equally likely. If the data signal is band limited to less than $1 / T \mathrm{~Hz}$, the spectrum of $w(t)$ will be band limited to less than $2 / T \mathrm{~Hz}$ and, so, must be of the form

$$
w(t)=A_{0}+A_{1} \sin \left(\frac{2 \pi t}{T}+\phi\right) .
$$

For a general $f(x), w(t)$ will have higher order harmonics.

The phase error function in the WDM is defined as

$$
p_{n}=w(n T+\tau)-w\left[\left(n+\frac{1}{2}\right) T+\tau\right]
$$

where $\tau$ is some arbitrary sampling phase. If frequency detection is desired, an additional quadrature error signal

$$
q_{n}=w\left[\left(n+\frac{1}{4}\right) T+\tau\right]-w\left[\left(n+\frac{3}{4}\right) T+\tau\right]
$$

is defined. In a phase-locked loop, $p_{n}$ is used to control the frequency of a VCO, and the feedback acts to force $p_{n}$ to zero. Fig. 1 shows the steady-state sampling phases for $p_{n}$ and $q_{n}$ on an eye diagram. It will be specified later how frequency detection is performed.

In a practical implementation, the expectation in (2) must be replaced by a time average, as in

$$
\hat{w}(t)=\frac{1}{K} \sum_{k=0}^{K-1} f(s(t-k T))
$$

where $K$ is the number of samples in the average. A sampleddata version of $\hat{w}(t)$, with sampling rate $1 / T$, can be computed using a transversal filter with $K$ taps, all of them of equal

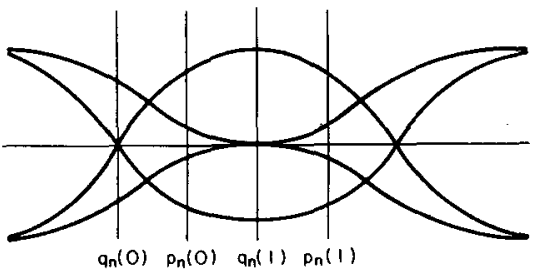

Fig. 1. Eye diagram and the steady-state sampling phase for $p_{n}$ and $q_{n}$.

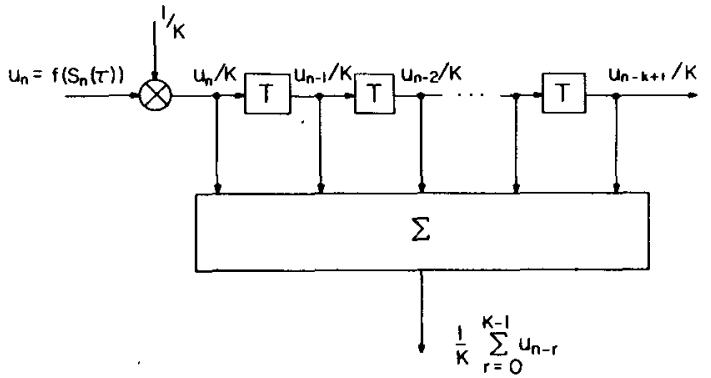

(a)

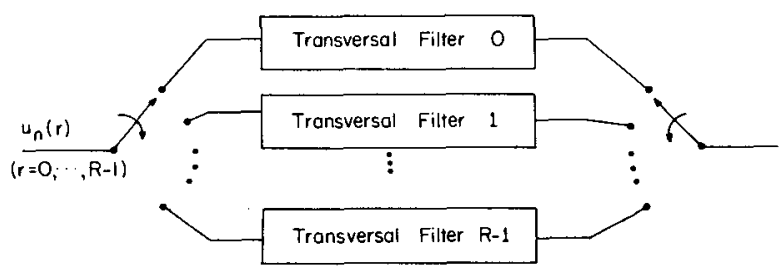

(b)

Fig. 2. (a) Averaging transversal filter with $K$ taps. (b) Time interleaved averaging filter.

weight $1 / K$ as shown in Fig. 2(a). In the figure, $u_{n}(\tau)$ are the samples of $f(s(t))$ taken at times $n T+\tau$ and $s_{n}(\tau) \stackrel{n}{=} s(n T+\tau)$. It is evident from (4) that aliasing will result from the aforementioned choice of sampling rate. The sampling rate can be increased to $R / T, R$ an integer, by using $R$ such transversal filters in a time interleaved fashion [Fig. 2(b)]. In this case $\tau$ is $r / R, 0 \leqslant r \leqslant R-1$. In the WDM, an oversampling factor $R=2$ is used, and the output samples of the two interleaved filters are subtracted as shown in Fig. 3(a). The subtractor function could also be located at the input of the transversal filters, in which case only one transversal filter is needed [Fig. $3(\mathrm{~b})$ ]. In general the output signal, the phase error estimate, is a slowly varying function of time. A high sampling rate is not necessary, and decimation by a large factor can be accepted. The storage requirements of the transversal filter are reduced accordingly. Fig. 3(c) shows a structure that decimates the signal by a factor $M$. In the limiting case $M=K$, no transversal filter is required at all.

Sometimes it may not be desirable to decrease the sampling rate excessively. One such case is when one wants to perform frequency detection, when the maximum frequency offset allowed in the VCO cannot be larger than half the sampling rate of the error signals to avoid aliasing. If a large pull-in range is desired in the $P L L$, a high sampling rate must be used for the error signals, and consequently, a longer transversal filter is required in Fig. 3(c). The storage requirements can be reduced while keeping the sampling rate high by using a recursive filter. Since in this application, accurate control of the bandwidth is not necessary, the coefficients of the filter can be approximated by numbers of the form $2^{-N}$ or $1-2^{-N}$ to avoid multipliers in the case of a digital implementation.

When oversampling by a factor $R=2$, neither of the two samples in each period will be taken at the instant of maximum eye opening after $P_{n}$ is driven to zero by the PLL. They 


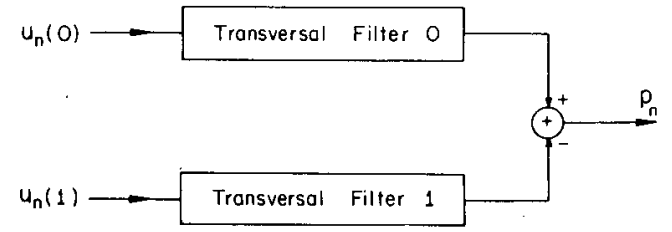

(a)

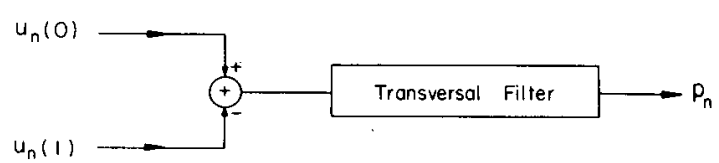

(b)

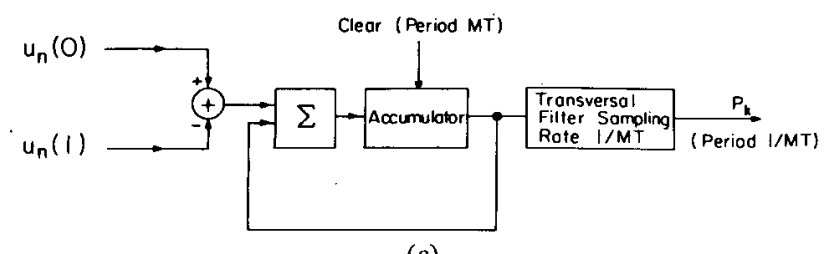

(c)

Fig. 3. (a) WDM using two interleaved filters. (b) WDM using one transversal filter. (c) WDM using one transversal filter with decimation.

will instead be located approximately (if the pulse is approximately symmetric) at $-T / 4$ and $T / 4$ relative to that point. Therefore, it seems that an oversampling factor of at least $R=4$ is needed, but this increase in $R$ is costly in receivers employing the EC method because the complexity of the echo canceler grows linearly with $R$. An approach which achieves an effective $R=4$, without increasing the sampling rate of the echo canceler, uses an interpolation filter at the output of the echo canceler to increase the effective oversampling factor. This filter must provide negligible distortion of the signal within the band $0 \leqslant f \leqslant 1 / T$, and a large alias suppression in the band $f>1 / T$. In order to satisfy these conditions, a relatively complicated filter is needed. A simpler solution to obtain an effective $R=4$ is the use of a linear phase all-pass network which approximates a delay $T / 4$. The resulting fractionally delayed samples can be used in the phase detector, so that one of the original samples will be located at the center of the eye. A second-order all-pass sect on with a transfer function

$$
H(z)=\frac{z^{-2}+c_{1} z^{-1}+c_{2}}{1+c_{1} z^{-1}+c_{2} z^{-2}}
$$

has been found to provide satisfactory results in computer simulations with

$$
\begin{aligned}
& c_{1}=0.429968 \\
& c_{2}=-0.048017
\end{aligned}
$$

Fig. 4 shows a typical example run for the case of a 2 mile gauge 26 line, with a 0.5 mile gauge 19 bridged tap at the center. In this example the sampling rate was $R=2$, but the output was computed 50 times with different values of the sampling phase; and the outputs plotted together so that the signal appears to be a continuous time signal. This was done to compare the pulse shapes before and after the phase shift network. For the same reason the output pulse was displaced in time by an amount equal to the delay of the network, namely $T / 4$. Although only one example is presented here, many more have been run, with similar or better results. We conclude that the use of this phase shift network provides a very simple and practical solution to the sample interpolation problem.

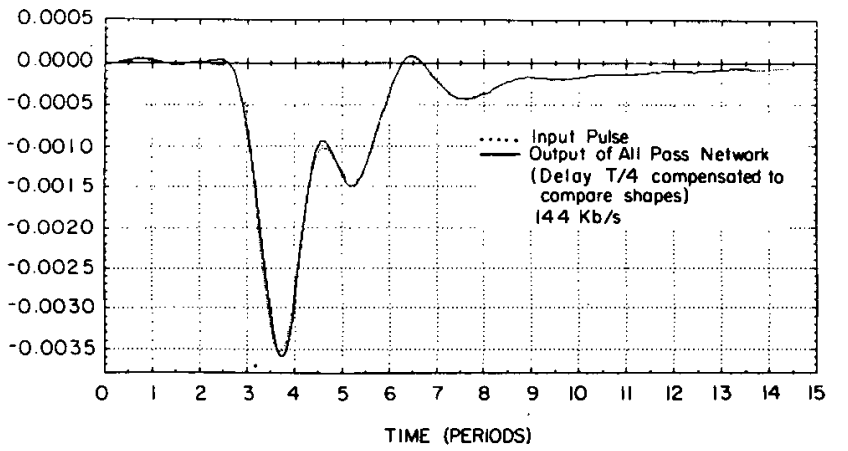

Fig. 4. All-pass filter approximation to a $T / 4$ delay: comparison of input and output with input appropriately delayed. For an ideal delay, the two waveforms would be identical.

\section{B. WDM Frequency Detector}

The WDM lends itself to the implementation, with little increase in complexity, of a frequency detector. This is potentially attractive because of the increase in the pull-in range of the PLL. In order to minimize jitter, very narrow loop bandwidth is required, which results in a limited pull-in range. This is no problem when accurate crystal-controlled VCO's are used, but the use of cheaper low-precision crystals, or even noncrystal VCO's, is an economically appealing possibility. The latter, in particular, would enable the monolithic integration of all the components of the VCO on the transceiver chip.

For frequency detection, an oversampling factor $R=2$ is not sufficient because aliasing distortion would not permit the distinguishing of positive and negative frequency offsets. The minimum oversampling factor depends on the maximum frequency offset allowed for the VCO. Since $R=4$ can be achieved without an increase in complexity of the echo canceler using the all-pass filter, assume $R=4$ in the subsequent analysis of the frequency detector.

The basic difference between a phase and a frequency detector is that the former measures the phase error modulo $T$, whereas the latter can keep track of cycle slips and, therefore, phase errors larger than $T$. The difference is illustrated in Fig. 5. Fig. 5(a) shows the characteristic of a phase detector, and Fig. 5(b) and (c) those of frequency detectors. In the case of Fig. 5 (b) the error characteristic is linear over a large number of cycles, whereas in Fig. 5(c), the characteristic saturates for phase errors $|\phi| \geqslant T / 2$. A way to make a phase detector into a frequency detector is to keep track of the number and the sign of the cycle slips. With an oversampling factor of $R=4$, the in-phase and quadrature error signals $p_{n}$ and $q_{n}$ defined in (5) and (6) can be used to detect these cycle slips.

A rotational detector [16] detects a cycle slip whenever the vector $\left(p_{n}, q_{n}\right)$ (Fig. 6) passes between the upper and the lower half-plane. The direction of the passage indicates whether the slip was positive or negative. Thus, a crossing from quadrant 1 to 4 or from 3 to 2 indicates a negative cycle slip, whereas a crossing from 4 to 1 or from 2 to 3 corresponds to a positive slip. The rotational detector lends itself to a simple implementation as shown in Fig. 7(a), and has been found to perform satisfactorily in computer simulations.

Another frequency detector is based on the quadricorrelator [16], as shown in Fig. 7(b). The quadricorrelator works on nearly the same principle as the rotational detector. The output of the hard limiter indicates whether the $\left(p_{n}, q_{n}\right)$ vector is in the upper or the lower half plane, and the derivative of $p_{n}$ indicates whether the vector is moving from the left to the right half-plane or vice versa. Thus, the sign of product of both signals represents the sign of frequency error (direction of rotation). The main difference is that the rotational detector counts only integral numbers of slips, whereas the quadricorrelator generates a proportional error signal. 


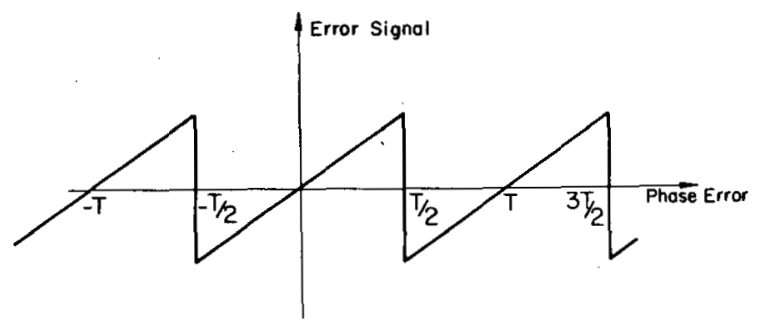

(a)

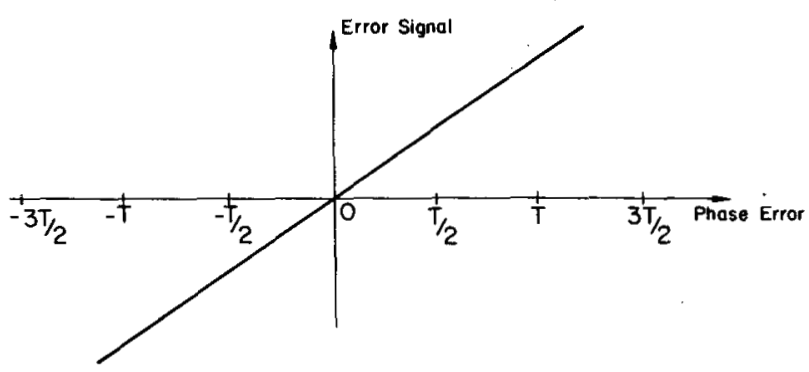

(b)

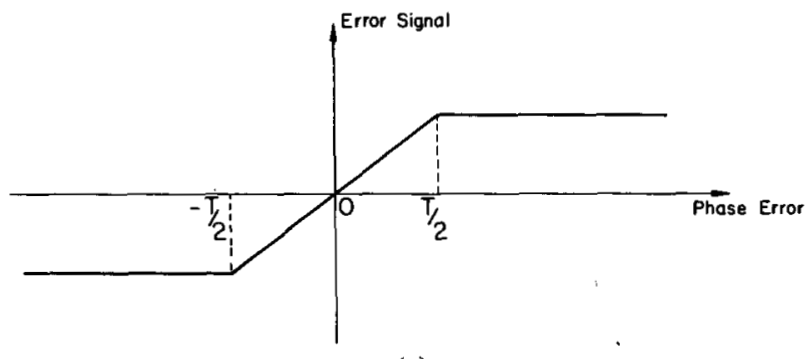

(c)

Fig. 5. (a) Characteristic of a phase detector. (b) Characteristic of a linear frequency detector. (c) Characteristic of a nonlinear frequency detector.

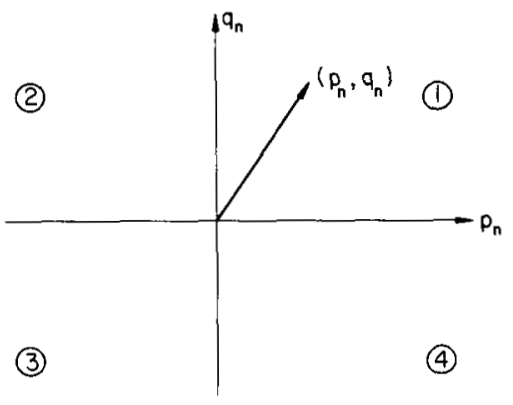

Fig. 6. Rotational detector detects a cycle slip whenever the vector $\left(p_{n} q_{n}\right)$ passes between the upper and lower half-plane

Results of computer simulations of both the rotational detector and the quadricorrelator, in the specific case of a subscriber loop receiver using WDM timing recovery, are reported in Section IV.

Unfortunately, any noncrystal VCO that can be integrated on a monolithic chip in MOS technology without trimming will have large errors in its center frequency. Errors of \pm 50 percent can be realistically expected. Frequency errors of this magnitude cannot be corrected with a continuously running frequency detector as described above. However, preliminary work indicates that it is possible to use a monolithic noncrystal VCO if an initial half-duplex startup sequence is used. During that sequence, pulses are sent from the central office at a much lower rate than the nominal, for example, 1/10T. A systematic sequence like $+1,-1,+1,-1,+1, \cdots$ is sent. At this low speed, all input filtering and equalization circuitry

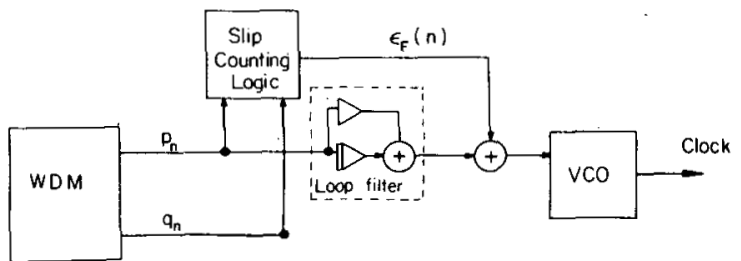

(a)

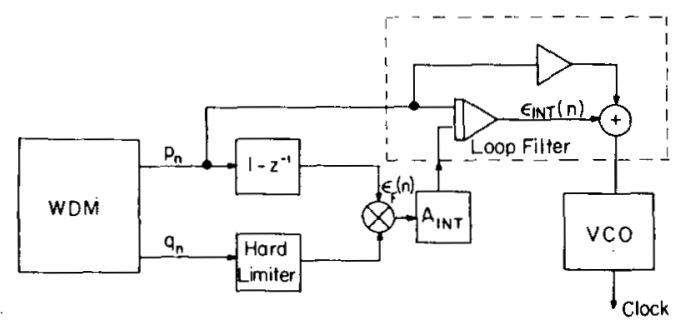

(b)

Fig. 7. (a) Rotational frequency detector. (b) Quadricorrelator frequency detector.

can be bypassed (obviously this circuitry needs an accurate clock, since it works on sampled data, so it cannot be used until the frequency of the clock has been adjusted). A simple threshold device generates a square wave from the received waveform, which can be used to adjust the VCO frequency using some of the standard digital frequency and phase detection techniques [6]. After frequency lock has been achieved, the operation is switched to full duplex and the WDM phase detector takes over the control of the VCO. The loop lock range must be large enough to allow tracking of frequency drifts caused by temperature variations during the operation of the VCO. Due to the large initial error in the VCO center frequency, a very large VCO dynamic range in frequency ${ }^{1}$ is required. This is of the order of $10^{6}: 1$, or about $20 \mathrm{bits}$. The complexity of an analog-digital implementation would be roughly equivalent to a 20 bit DAC, although it seems that if appropriate interpolation techniques are used, the system can be implemented in a reasonable amount of silicon area. Since frequency lock is achieved in this case during the startup sequence, another continuously running WDM frequency detector is not required.

In summary, frequency detection may be advantageous whenever a lower accuracy free-running frequency for the $\mathrm{VCO}$ is desired, as would be obtained from using a cheaper crystal. If monolithic noncrystal VCO's are used, a continuously running WDM frequency detector does not provide enough pull-in range, and frequency acquisition must be achieved during an initial startup sequence.

\section{Timing Tone and Jitter Analysis}

In this section, closed form expressions for the power of the timing tone and the jitter generated by pulse overlap in WDM are derived.

Consider a data signal $s(t)$ as in (1). If the channel is possibly nonlinear and its impulse response has finite duration $N T$, where $N$ is the number of periods over which the channel impulse response is nonzero, it is shown in [1] that the signal can be represented as

$$
s_{n}(r)=s\left(n T+\frac{r T}{R}\right)=\boldsymbol{x}_{n} T \cdot h(r)
$$

' In a digitally controlled VCO, the frequency can be adjusted only in steps. Dynamic range of the VCO is defined as the ratio of the total tuning range to the smallest frequency step that can be generated. 
where

$$
\begin{aligned}
\boldsymbol{x}_{n}= & \left(1, x_{n}, x_{n-1}, \cdots, x_{n-N+1}, x_{n} x_{n-1}, x_{n} x_{n-2},\right. \\
& \left.\cdots, x_{n-N+2} x_{n-N+1}, \cdots, x_{n} x_{n-1}, \cdots, x_{n-N+1}\right)^{T}
\end{aligned}
$$

is the $2^{N}$-dimensional "augmented transmitted symbol vector" (assuming the data symbols are binary), and

$$
\begin{aligned}
\boldsymbol{h}(r)= & \left(h_{0}, h_{1}(0, r), h_{1}(1, r), \cdots, h_{1}(N-1, r),\right. \\
& \cdots, h_{2}(0,1, r), h_{2}(0,2, r), \cdots, h_{2}(N-2, N-1, r), \\
& \left.\cdots, h_{N}(r)\right)^{T}
\end{aligned}
$$

is the $2^{N}$-dimensional nonlinear channel impulse response vector.

The notation in (10) allows for nonlinearities in the transmission channel, although in practice most channels are-linear or very approximately linear. This notation, nevertheless, is useful to represent the nonlinear operation deliberately performed on the signal in order to generate a timing tone. If the function $f(\cdot)$ is such a nonlinear operation, it is shown in [1] that $f\left(s_{n}(r)\right)$ can be expressed as

$$
u_{n}(r)=f\left(s_{n}(r)\right)=\boldsymbol{x}_{n}^{T} \cdot \boldsymbol{F}[\boldsymbol{h}(r)]=\boldsymbol{x}_{n}{ }^{T} \cdot g(r)
$$

where $\boldsymbol{F}[\boldsymbol{h}(r)]$ is a $2^{N}$-dimensional nonlinear transformation induced by $f(\cdot)$ on the vector $h(r)$ and

$$
g(r)=F[h(r)]
$$

In Appendix A, a method to compute $\boldsymbol{g}(r)$ for a given $h(t)$ and $f(\cdot)$ is shown.

If the samples $u_{n}(r)$ are placed at the input to $R$ time interleaved filters with unit sample responses $\nu_{r}(k),(0 \leqslant r<R)$, as in Fig. 2(b), Appendix B shows that the power of the output signal is

$$
E\left\{y_{n}^{2}(r)\right\}=\alpha_{0}(r)\left|g_{0}(r)\right|^{2}+g^{T}(r) M g(r)
$$

where

$$
\alpha_{0}(r)=\left(\sum_{k=-\infty}^{\infty} \nu_{r}(k)\right)^{2}
$$

and $M$ is a $2^{N}$-dimensional square matrix which depends on the filter response. The first term in (15) represents the power of the timing tone at the output of the rth filter, whereas the second term represents the jitter power, or the variance of the timing signal.

Not all practical filters can be represented by an interleaved structure as in Fig. 2(b). However, one important case which can is when the unit sample response of the filter (at a sampling rate $R / T$ ) can be expressed as

$\nu(r+n R)=P(n) Q(r)$.

The response of this filter for an input $\lambda(n)$ is

$$
\begin{aligned}
y(n) & =\sum_{m} \sum_{r=0}^{R-1} \nu(r+m R) \lambda(n-m R-r) \\
& =\sum_{m} \sum_{r=0}^{R-1} P(m) Q(r) \lambda(n-m R-R) \\
& =\sum_{m} P(m) z(n-m R) .
\end{aligned}
$$

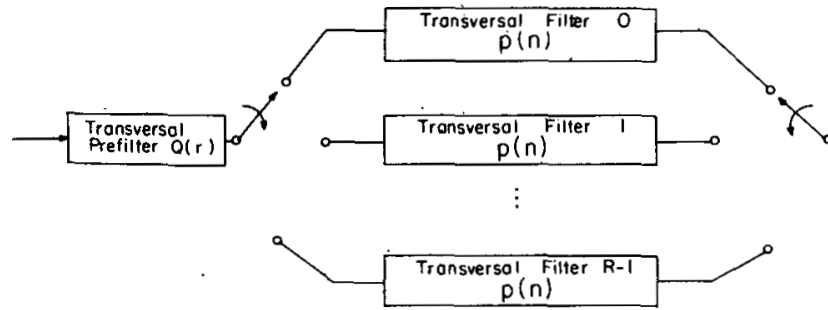

Fig. 8. Interleaved structure of a filter with unit sample response expressed as $\nu(r+n R)=\dot{P}(n) Q(r)$.

This can be represented by a transversal prefilter with unit sample response $Q(r),(0 \leqslant r \leqslant R)$ and sampling rate $R / T$, followed by $R$ interleaved finite impulse response (FIR) transversal filters with the same responses $P(m)$ and sampling rates $1 / T$ (Fig. 8 ). Sometimes the output can be decimated, and not all the $R$ interleaved FIR filters are required. One example of a filter of this kind is the WDM filter. If only phase detection is performed, $R=2$ and

$$
\begin{array}{ll}
Q(r)=(-1)^{r}, & r=0,1 \\
P(n)=\frac{1}{K}, & 0 \leqslant n \leqslant K-1 \\
P(n)=0, & n<0 \text { or } n \geqslant K
\end{array}
$$

and if frequency detection is needed, $R=4$ and

$$
\begin{array}{ll}
Q(r)=(-1)^{r / 2}, & r=0 \text { or } r=2 \\
Q(r)=0, & r=1 \text { or } r=3 \\
P(n)=\frac{1}{K} ; & 0 \leqslant n<K \\
P(n)=0, & n<0 \text { or } n \geqslant K .
\end{array}
$$

For phase detection only one of the $R$ interleaved FIR filters is needed. For frequency detection we need two, to generate the in-phase and quadrature signals: For the WDM filters, (15) is still valid if we replace $g(r)$ by $g(0)-g(1)$ in the case of phase detection alone, and by $\boldsymbol{g}(0)-\boldsymbol{g}(2)$ and $\boldsymbol{g}(1)-\boldsymbol{g}(3)$, respectively for the in-phase and quadrature signals in the case of phase and frequency detection. The appropriate $\boldsymbol{M}$ matrix is computed in Appendix B.

For the sake of comparison, it is interesting to consider the case when the filter is a resonator with unit sample response

$$
\nu(n)=e^{-\alpha n / R} \sin \left(\frac{\omega n}{R}\right) .
$$

This case is slightly more involved because the filter does not have the structure of (17). However, it can be split into two filters with responses

$$
\nu_{1}(n)=e^{-(\alpha-j \omega) n / R}
$$

and

$$
\nu_{2}(n)=e^{-(\alpha+j \omega) n / R},
$$

each with a structure as in (17), and the outputs combined. The results are further simplified if we consider a resonator centered at the data rate, that is, $\omega=2 \pi$. The details of the 
calculation are left to the reader, but the final result is

$$
\begin{aligned}
E\left\{y_{n}{ }^{2}(r)\right\}= & \alpha_{0}\left|G_{0}(2 \pi)\right|^{2}+\alpha_{0} \operatorname{Im}\left(G_{0}{ }^{2}(2 \pi) e^{-j 4 \pi r / R}\right) \\
& +G(-2 \pi) M G(2 \pi)+\operatorname{Im}[G(2 \pi) M G(2 \pi) \\
& \left.\cdot e^{-i 4 \pi r / R}\right]
\end{aligned}
$$

where $\alpha_{0}$ and $M$ are defined in Appendix B, and $G(\omega)$ is the discrete Fourier transform of vector $g(r)(0 \leqslant r \leqslant R-1)$. The first two terms in (24) represent the timing tone, and the last two represent the jitter.

This discrete time resonator can be easily generated to continuous time by letting $R \rightarrow \infty$, and then can be used to compute the jitter performance of the spectral line method. In computer simulations (24) was used with a large value of $R(R=100)$.

Using these results, the jitter was evaluated for a number of practical cable configurations whose pulse responses were obtained from a computer line modeling program. The results are shown in Table II and further described in Section III.

\section{Comparison of the WDM to the Spectral Line Method}

In the spectral line method, the timing tone obtained after the nonlinear operation on the data signal is recovered by a narrow-band filter. Instead of using a bandpass filter centered at the data rate, a low-pass filter of equivalent bandwidth can be used if the filtering operation is preceded by a frequency translation of the spectrum down to $\mathrm{dc}$. This frequency conversion can be achieved by multiplication of the timing signal by a sinusoidal reference signal with frequency nominally equal to the data rate. This is the operation usually performed when the timing tone is filtered with a phase-locked loop, where the reference signal is the output of the VCO. Any departure of the frequency of the reference signal from its nominal value will create a beat tone at the output of the lowpass filter which can be used to control the VCO that generates the reference signal. If the timing tone is $A \sin (\omega t+\phi)$ and the reference signal is $\sin \left(\omega_{r} t\right)$, demodulation will create the tones $\frac{1}{2} \cos \left(\left(\omega-\omega_{r}\right) t+\phi\right)$ and $-\frac{1}{2} \cos \left(\left(\omega+\omega_{r}\right) t+\phi\right)$.

The demodulation of the timing signal can be performed in continuous time or in sampled data fashion. Assume in the latter that the sampling rate is exactly twice the frequency of the reference signal. Then, if the departure of $\omega_{r}$ from its nominal value $\omega$ is smaller than $\omega_{p}$, the frequency difference tone will be adequately represented without aliasing distortion. The sum frequency tone will be aliased down to $\frac{1}{2} \cos \left(\omega-\omega_{r}\right) t$, and will reinforce the difference frequency tone. Clearly, it is not possible to distinguish whether the frequency error is positive or negative, and therefore, no frequency detection is possible with a sampling rate twice the reference frequency. With the sampler locked to the VCO as assumed here, the samples of the reference signal have only two values $+\sin \psi$ and $-\sin \psi$, where $\psi$ is the relative phase between the sampler and the VCO. If $\psi=\pi / 2$, the samples are +1 and -1 . The phase detector is then effectively computing the difference between the even and odd-order samples of the timing signal. If, in addition, the low-pass filter is an averaging filter (that is, the output samples are computed as the average of a certain number $K$ of input samples), this sampled-data version of the spectral line technique becomes exactly equivalent to the WDM as described in Section II-A.

So far we have considered that the timing signal consists of a purely sinusoidal tone at the data rate, with no harmonics and no continuous spectral components. When the nonlinear operation performed on the data signal is a square-law operation, no harmonics of the discrete timing tone are generated, but there are continuous spectral components. If the nonlinear operation is other than a square-law operation, there will be both higher order harmonics of the timing tone and continu- ous components. The WDM is no longer exactly equivalent to the spectral line technique because the phase error signal will include the effect of the dc aliases of the higher order harmonics of the timing tone, and the phase recovered by the WDM is then different from the phase recovered by the spectral line technique. Furthermore, the jitter is expected to be different even in the case of a square-law operation, because of the aliasing of continuous components above the data rate. We will show in the next section that the WDM generally performs better than the spectral line method.

\section{Performance of the WDM}

The performance of the WDM when operating on real telephone lines has been studied using channel impulse responses computed by a cable modeling program [17]. This program models the line sections, gauge discontinuities, bridged taps, transformers, transmit and receive filters, and an equalizer. The transmit and receive filters used were all-pole minimal intersymbol interference filters [18], and the equalizer frequency response was

$$
F(s)=\left(\frac{b}{a}\right)\left(\frac{s+a}{s+b}\right)
$$

where $a$ and $b$ are the zero and the pole locations. (Usually $b$ is fixed and $a$ is adjustable.)

A large number of cable configurations were analyzed to determine how cable imperfections affect the system performance. Although the amount of pulse distortion would increase with more than one bridged tap, only lines with single bridged tap are considered here. More study is needed to assess the validity of the WDM in cases with more than one bridged tap. Model program output showed that the transmission path (unlike the echo-path) impulse response is almost completely insensitive to the location of the bridged tap, and thus, a center location was assumed. The reflection originates at the open end of the bridged tap, and the longer the tap, the longer the delay of the reflection with respect to the main pulse, and the smaller its relative height.

The pulse distortion generated by a bridged tap depends on the transmit and receive filters used. Severe band limiting of the signal causes the reflection from the bridged tap to merge with the main pulse. The only observable effect may be simply a widening of the pulse, particularly for lower data rates, for example, $80 \mathrm{kbits} / \mathrm{s}$ and a gauge 26 bridged tap. When the data rate is increased, causing a corresponding increase in the bandwidth of the transmit and receive filters, the reflections from bridged taps start to be resolved, causing more concern about the phase of the recovered timing. Clearly, the intensity of the reflection is also strongly dependent on the bridged tap gauge. Gauge 26 bridged taps have been found in the modeling to cause very little observable effect in band-limited systems, even at data rates as high as $144 \mathrm{kbits} / \mathrm{s}$. Because of the larger instantaneous data rates involved, TCM systems may be more susceptible to sampling phase offsets caused by bridged taps.

The typical configuration presented here consists of a 2 mile gauge 26 cable with a bridged tap at the center. The wire gauge of the bridged tap is 19 and its length is varied from 0.1 to 0.5 miles. A gauge 19 bridged tap was used in order to minimize the attenuation of the reflected wave and, thus, present the worst case. Fig. 9(a) shows the impulse response of the five different cables considered at a data rate of $144 \mathrm{kbits} / \mathrm{s}$, whereas Fig. 10(a) shows the same response at $256 \mathrm{kbits} / \mathrm{s}$.

Another computer program was written to evaluate timing function (1). It was assumed that the impulse length was $5 T$, and consequently, the average was computed over the 32 possible sets of overlapping data bits. The sampling phase recovered 


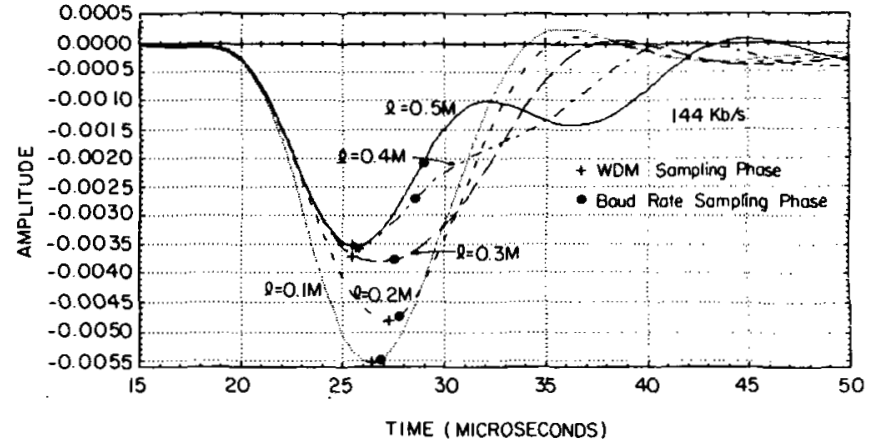

(a)

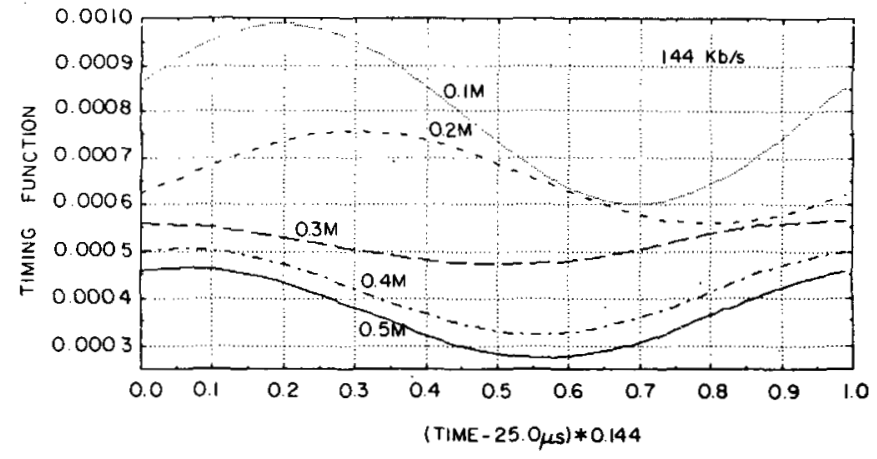

(b)

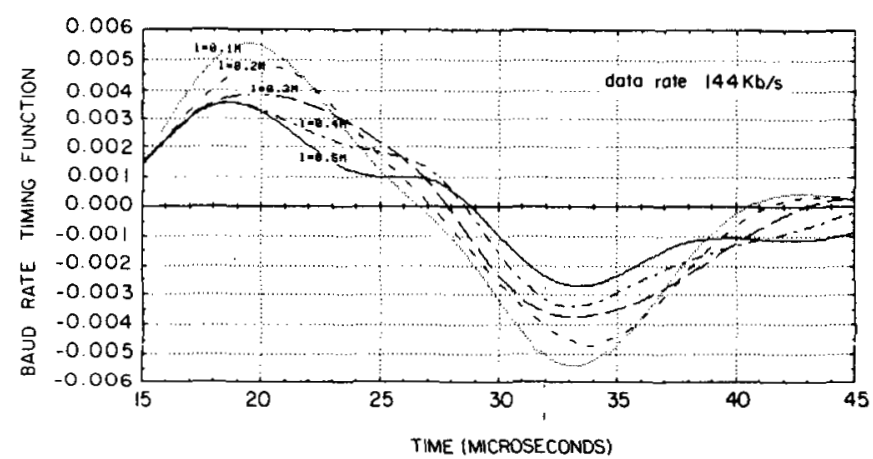

(c)

Fig. 9. (a) Timing phase obtained by WDM and the baud rate sampling technique at $144 \mathrm{kbits} / \mathrm{s}$. (b) Timing function of WDM at $144 \mathrm{kbits} / \mathrm{s}$. (c) Timing function of the baud rate sampling technique at $144 \mathrm{kbits} / \mathrm{s}$.

by the WDM was found solving the equation

$$
w\left(t-\frac{T}{4}\right)=w\left(t+\frac{T}{4}\right) .
$$

This phase is shown in Figs. $9(\mathrm{a})$ and $10(\mathrm{a})$, whereas the timing function is shown in Fig. 9(b) for $144 \mathrm{kbit} / \mathrm{s}$ transmission, and in Fig. 10(b) for $256 \mathrm{kbit} / \mathrm{s}$ transmission. The phase recovered by the WDM is very close to the maximum of the pulse even in the presence of strong bridged tap distortion.

Another timing recovery technique, the baud rate sampling technique [7], would allow a further simplification of an echo canceler. For symmetric received pulses, the sampling phase determined by this technique is the same as that of WDM and coincides with the maximum of the pulse. However, when the phase determined by the baud rate technique employing the timing function

$$
w(t)=h(t-T)-h(t+T)
$$

was calculated, a strong dependence on pulse shape was ob-

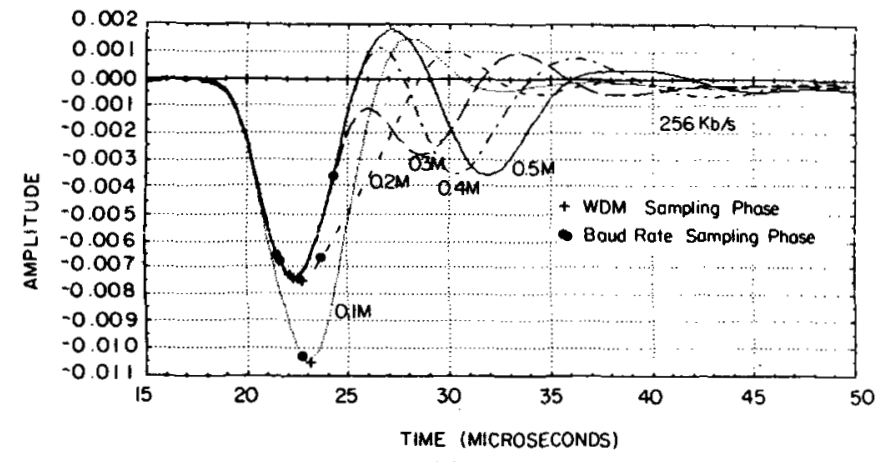

(a)

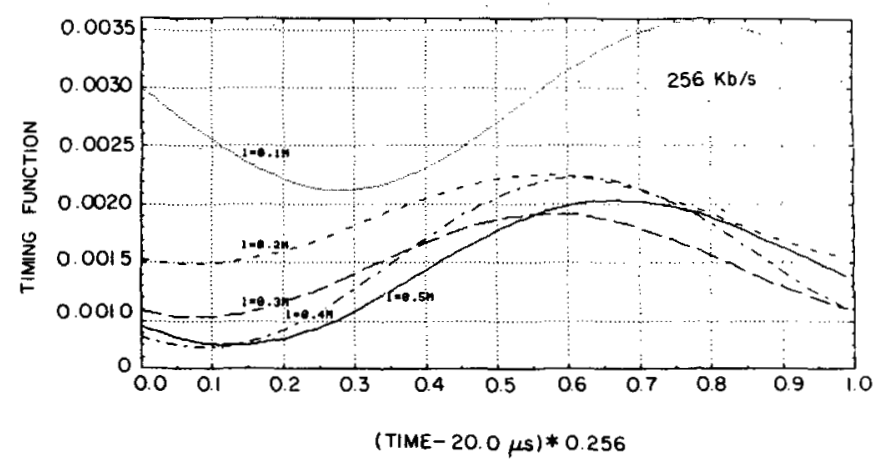

(b)

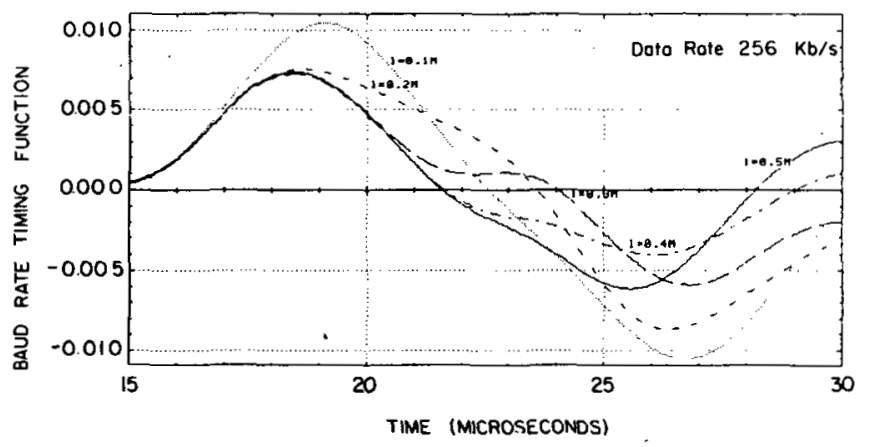

(c)

Fig. 10. (a) Timing phase obtained by WDM and the baud rate sampling technique at $256 \mathrm{kbits} / \mathrm{s}$. (b) Timing function of WDM at $256 \mathrm{kbits} / \mathrm{s}$. Timing function of the baud rate sampling technique at $256 \mathrm{kbits} / \mathrm{s}$.

TABLE 1

SAMPLING EPOCH OF THE TIMING RECOVERY SYSTEMS

\begin{tabular}{|c|c|c|c|c|}
\hline \multicolumn{3}{|c|}{ Sampting Epoch of Timing Recovery System } \\
\hline $\begin{array}{c}\text { Bridged ToD } \\
\text { Length }\end{array}$ & \multicolumn{2}{|c|}{ WDM } & \multicolumn{2}{|c|}{ Boud Rate Sompling } \\
\cline { 2 - 5 } & $144 \mathrm{~Kb} / \mathrm{s}$ & $256 \mathrm{~Kb} / \mathrm{s}$ & $144 \mathrm{~Kb} / \mathrm{s}$ & $256 \mathrm{~Kb} / \mathrm{s}$ \\
\hline 0.1 Mile & $26.39 \mu \mathrm{s}$ & $23.08 \mu \mathrm{s}$ & $26.60 \mu \mathrm{s}$ & $22.54 \mu \mathrm{s}$ \\
\hline 0.2 & $27.08 \mu \mathrm{s}$ & $22.26 \mu \mathrm{s}$ & $27.15 \mu \mathrm{s}$ & $23.59 \mu \mathrm{s}$ \\
\hline 0.3 & $25.35 \mu \mathrm{s}$ & $2230 \mu \mathrm{s}$ & $27.84 \mu \mathrm{s}$ & $24.02 \mu \mathrm{s}$ \\
\hline 0.4 & $25.71 \mu \mathrm{s}$ & $22.42 \mu \mathrm{s}$ & $28.54 \mu \mathrm{s}$ & $21.64 \mu \mathrm{s}$ \\
\hline 0.5 & $25.37 \mu \mathrm{s}$ & $22.69 \mu \mathrm{s}$ & $28.82 \mu \mathrm{s}$ & $21.56 \mu \mathrm{s}$ \\
\hline
\end{tabular}

served. The timing functions for the five different configurations are shown in Figs. 9(c) and 10(c), and the corresponding sampling phases, computed as the solutions of $w(t)=0$, are shown in Figs. 9(a) and 10(a). The sampling phases of both WDM and baud rate sampling are also summarized in Table 1. For cases of severe pulse distortion, for example, when the bridged tap length is 0.4 or 0.5 miles, the baud rate sampling technique yields a completely erroneous sampling phase un- 
less line coding is modified from the binary or AMI code considered here. However, even with binary or AMI coding, baudrate sampling is not ruled out completely, since computer simulations showed that in band-limited systems operating on lines with gauge 26 bridged taps, the pulse distortion is negligible, and thus, baud rate timing recovery may be appropriate. $^{2}$

The final conclusion on baud rate timing recovery requires a statistical study of the telephone lines deployed in the field, to determine the percentage on which the system can be expected to work properly. If this information is not available, a more conservative approach like the WDM seems advisable.

The jitter performance of WDM when operating on the same example lines was studied using the theory developed in Section II, which applies directly to binary line coding. For comparison, the jitter performance of the continuous time spectral line method was also computed using nonlinear functions $x^{2},|x|$, and $x^{4}$. In order to make the examples more directly comparable, the averaging filter of the WDM was replaced here by a recursive filter with a unit sample response $e^{-\alpha n}$, while in the spectral line method a resonator centered at the data rate, with an impulse response $e^{(-\alpha+j \omega) t}$ was used. The transmission speed was $144 \mathrm{kbits} / \mathrm{s}$, and the filter bandwidth was in both cases $B=\alpha / 2 \pi=3.6 \mathrm{~Hz}$. The jitter power was computed after adjusting the signal level to yield a normalized timing tone amplitude with a unity peak value in order to compare with the results of the PLL simulation of Section IV. The PLL bandwidth depends on the phase detector gain, which in turn depends on the timing tone intensity. If $T$ is also normalized to 1 , the jitter power can be expressed in decibels, and directly compared to the output of the PLL in Section IV.

The jitter power for WDM is given by

$$
P=\pi B T\left(g^{T}(1)-g^{T}(2)\right) M(g(1)-g(2))
$$

and for the spectral line by the two last terms of (24). A computer program read the line impulse response and computed the Hadamard matrix (Appendix A), $\boldsymbol{g}(r), C(m)$, and finally the jitter. The results are shown in Table II. The WDM yields a performance comparable or superior to the spectral line method for most cable configurations. The absolute value function in the latter not only is the easiest to implement, but also gives the best results in most cases. The worst performance is associated with bridged taps of an intermediate length, such that the delay of the reflected pulse is about $T / 2$. When the delay increases to $T$, the jitter decreases.

\section{An Example of Timing Recovery Design}

In this section the conclusions of the previous sections are applied to the practical design of the timing extraction block of a subscriber loop receiver. Both phase and frequency detection are used. For the latter, one of the examples uses a rotational detector, and the other a quadricorrelator. A proportional-plus-integral (PI) loop filter for the phase error signal and an integral-only filter for the frequency error are used, as recommended in [16]. In the case of the rotational detector, the integral of the frequency error is computed simply by counting slips, a technique that introduces a very coarse quantization in the filter output signal. This is advantageous since, once in lock, the frequency detector does not disturb the phase-locked loop. In the quadricorrelator, the frequency detector is always active, even in lock, and the fluctuations of the frequency error signal introduce an extra jitter in the recovered clock.

\footnotetext{
${ }^{2}$ If equalization is performed at a sampling rate $R=2$, sampling phase is noncritical. However, for this case there is no strong motivation to use baud rate timing recovery.
}

TABLE II

TIMING TONE TO JITTER POWER RATIO IN DECIBELS WITHOUT FREQUENCY DETECTOR

\begin{tabular}{|c|c||c|c|c|c|c|}
\hline \multicolumn{2}{|c|}{} & $0.1 \mathrm{M}$ & $0.2 \mathrm{M}$ & $0.3 \mathrm{M}$ & $0.4 \mathrm{M}$ & $0.5 \mathrm{M}$ \\
\hline \multirow{3}{*}{ WDM } & $x^{2}$ & 61.79 & 64.08 & 4645 & 58.64 & 54.05 \\
\cline { 2 - 7 } & $|\mathrm{X}|$ & 62.42 & 64.71 & 4723 & 57.91 & 54.16 \\
\cline { 2 - 7 } & $x^{4}$ & 61.12 & 61.69 & 5086 & 54.40 & 5428 \\
\hline \multirow{3}{*}{$\begin{array}{l}\text { Spectral } \\
\text { Line }\end{array}$} & $x^{2}$ & 5736 & 57.01 & 53.35 & 59.59 & 61.43 \\
\cline { 2 - 7 } & $|x|$ & 55.96 & 56.00 & 51.79 & 58.76 & 59.27 \\
\cline { 2 - 7 } & $x^{4}$ & 60.83 & 61.33 & 58.29 & 58.85 & 6347 \\
\hline
\end{tabular}

Block diagrams of the two approaches are shown in Fig. 7 (a) and (b). The sampling rate of both the in-phase and the quadrature error signals $p$ and $q$ was chosen equal to $1 \mathrm{kHz}$ in order to allow relatively large initial offsets in the VCO. Clearly, the offset cannot be larger than the sampling rate of the error signals. Prior to the decimation of $p$ and $q$, a low-pass filtering was performed using a recursive first-order filter with a unit sample response $e^{-\alpha n}$. The band width was $B=2 \pi \alpha / T=$ $100 \mathrm{~Hz}$ in the case of the rotational detector, and $B=500 \mathrm{~Hz}$ in the case of the quadricorrelator.

The complete timing recovery systems were simulated for the same five example lines considered in Section III. Simulations for both binary and AMI line codes indicated that the jitter performance is quite similar, and only results for the AMI case are presented here. The convergence transients for an initial VCO offset of $2000 \mathrm{ppm}$ or $288 \mathrm{~Hz}$ are shown in Figs. 11 (a) and (b) and 12(a) and (b) for the rotational detector and the quadricorrelator; Fig. 11 (a) and (b) shows the phase and Fig. 12(a) and (b) the output of the phase detector. Only one case, corresponding to a 0.5 mile $B T$, is shown here, but similar results were obtained for the other cases. Lock was acquired by the rotational detector in less than 14400 cycles, which corresponds to $100 \mathrm{~ms}$, in all the cases. A tradeoff between speed of acquisition and residual jitter exists in the case of the quadricorrelator. Using a large gain in the frequency loop, acquisition can be speeded, but the extra jitter introduced by the frequency error signal under lock conditions is higher. In the simulations shown here, a longer acquisition time than was obtained for the rotational detector was deliberately accepted to decrease somewhat the steady-state jitter. However, it seems that the overall performance is poorer, and thus, the rotational detector is to be preferred.

The steady-state phase after lock coincides with that shown in Fig. 9(a) for the WDM. The jitter performance was also simulated and the results, shown in Table III, are seen to agree closely with the analytical results of Table II for the case of the rotational detector, and are significantly worse in the case of the quadricorrelator.

\section{CONCLUSIONS}

The use of discrete-time timing recovery techniques has been found to be a viable alternative to conversion of the data signal to continuous time followed by continuous time-timing recovery. The former is a preferable approach for realization with MOS monolithic technology. Of particular interest is the WDM, which has been studied both analytically and by computer simulation and found to be as good or better than its continuous-time counterpart, the spectral line method. Computer simulations have shown that the recovered phase is very satisfactory even in the presence of severe pulse asymmetry due to bridged taps.

Alternatives for the implementation of frequency detectors have also been discussed. The advantage of adding a frequency detector in addition to the phase detector is an increased pullin range. This could allow a less accurate free-running frequency for the VCO. 


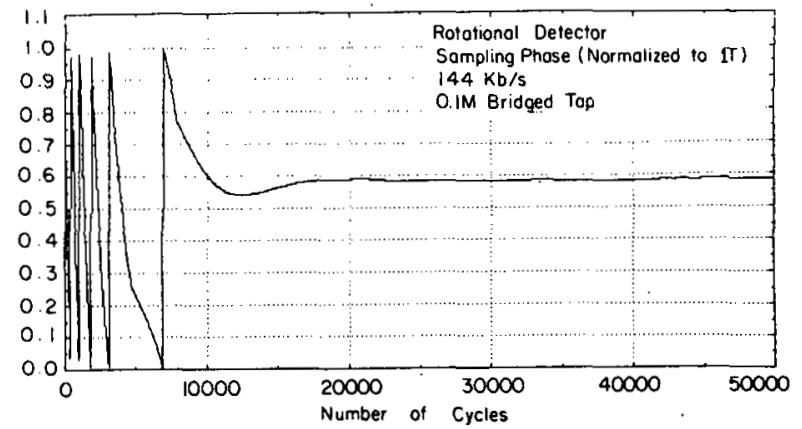

(a)

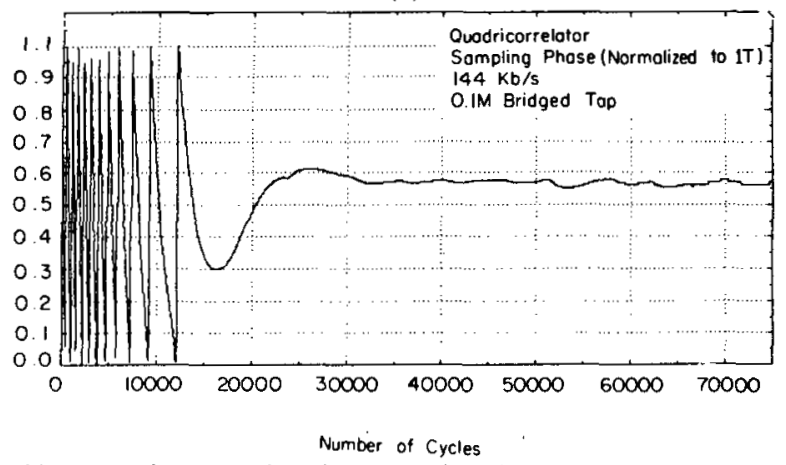

Fig. 11. Transient behavior of the sampling phase acquired by the rotational detector. (b) Transient behavior of the sampling phase acquired by the quadricorrelator detector.

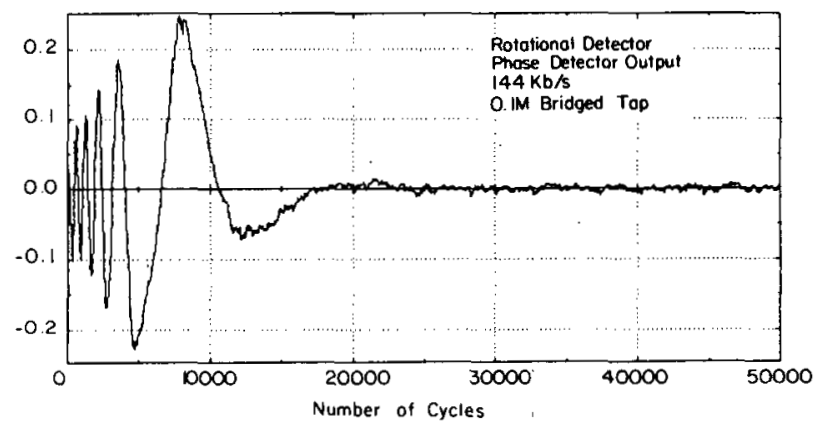

(a)

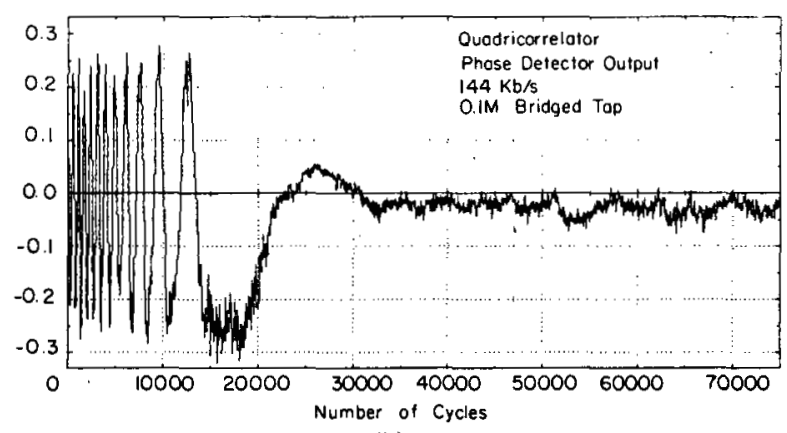

(b)

Fig. 12. (a) Phase detector output of the rotational detector versus time. (b) Phase detector output of the quadricorrelator detector versus time.

TABLE III

TIMING TONE TO JITTER POWER RATIO IN DECIBELS WITH FREQUENCY DETECTOR

\begin{tabular}{|l|c|c|c|c|c|}
\hline & $0.1 \mathrm{M}$ & $0.2 \mathrm{M}$ & $0.3 \mathrm{M}$ & $0.4 \mathrm{M}$ & $0.5 \mathrm{M}$ \\
\hline $\begin{array}{l}\text { rotational } \\
\text { detector }\end{array}$ & 62.52 & 64.56 & 46.41 & 55.56 & 52.85 \\
\hline $\begin{array}{l}\text { quadri - } \\
\text { correlctor }\end{array}$ & 42.98 & 40.35 & 31.08 & 44.16 & 40.87 \\
\hline
\end{tabular}

Finally, a complete implementation of a WDM timing recovery circuit has been proposed. The sampling rate is twice the data rate, thus permitting the use of a relatively simple echo canceler, but a second-order all-pass linear phase network is used to generate a $T / 4$ delayed version of each sample, increasing the effective sampling rate to four times the data rate. At this sampling rate both frequency and phase detection can be performed.

At present some additional work is being performed on modifying the line coding to improve the performance of the baud rate sampling technique, and the results will be reported in a future paper.

\section{APPENDiX A}

It has been shown [2] that a nonlinear function of $N$ bits $f\left(z_{0}, z_{1}, \cdots, z_{N-1}\right)$ can be expanded in the form ${ }^{1}$

$$
\begin{aligned}
& f\left(z_{0}, z_{1}, \cdots, z_{N-1}\right) \\
& =c_{0}+\sum_{k=0}^{N-1} c_{1}(k) z_{k}+\sum_{k_{1}, k_{2}=0}^{N-1} c_{2}\left(k_{1}, k_{2}\right) z_{k_{1}} z_{k_{2}} \\
& \quad+\cdots+c_{N} z_{k_{1}} z_{k_{2}} \cdots z_{k_{N}} .
\end{aligned}
$$

A simple technique was given in [1] to compute the coefficients $c_{0}, c_{1}(k), \cdots, c_{N}$ when the bits $z_{k}$ can assume the values 0 and 1 . In the more practical case when the $z_{k}$ assume values +1 and -1 , the only way to compute the coefficients of (A-1) is to solve a system of $2^{N}$ equations with $2^{N}$ unknowns (the coefficients). Although it may seem that solving that system is cumbersome, it is actually trivial, because the matrix of the system is orthogonal, and in a convenient representation, it is also symmetric, so that it is its own inverse.

In the inner product notation of (10), expansion (A-1) can be expressed

$$
f\left(z_{0}, z_{1}, \cdots, z_{N-1}\right)=z^{T} \cdot c .
$$

Define a $2^{N}$-dimensional vector $f$ whose components are the values of the nonlinear function $f$ for all the $2^{N}$ combinations of the variables $z_{0}, \cdots, z_{N-1}$ :

$$
f=\left[\begin{array}{c}
f(+1,+1,+1, \cdots,+1) \\
f(-1,+1,+1, \cdots,+1) \\
f(+1,-1,+1, \cdots,+1) \\
f(+1,+1,-1, \cdots,+1) \\
\vdots \\
f(-1,+1,-1, \cdots,+1) \\
\vdots \\
f(+1,+1,+1, \cdots,-1) \\
\vdots \\
f(-1,+1,+1, \cdots,-1) \\
\vdots \\
f(-1,-1,-1, \cdots,+1) \\
f(-1,-1,-1, \cdots,-1)
\end{array}\right]
$$

When the vectors $z$ are also formed for all the combinations 
of values +1 and -1 of the binary. variables $z_{k}$, a set $B$ of $2^{N}$ vectors $z_{1}, \cdots, z_{2} N$ is obtained. We will show that $B$ is an orthogonal basis of the $2^{N}$-dimensional vector space $R^{2}$ (the space of all $2^{N}$-tuples of real numbers). To prove this, consider any two vectors $\boldsymbol{x}$ and $\boldsymbol{y}$ in $\boldsymbol{B}$ and form their inner product:

$$
\begin{aligned}
\boldsymbol{x} \cdot \boldsymbol{y}= & 1+x_{0} y_{0}+x_{1} y_{1}+\cdots+x_{N-1} y_{N-1} \\
& +\cdots+x_{0} x_{1} \cdots x_{N-1} y_{0} y_{1} \cdots y_{N-1} \\
= & \left(1+x_{0} y_{0}\right)\left(1+x_{1} y_{1}\right) \cdots\left(1+\dot{x}_{N-1} y_{N-1}\right) .
\end{aligned}
$$

It is clear that this inner product is nonzero only if $x_{k}=y_{k}$ for all $k=0, \cdots, N-1$, which occurs only if $\boldsymbol{x}=\boldsymbol{y}$. Any two vectors in $\boldsymbol{B}$ are orthogonal, and $\boldsymbol{B}$ is an orthogonal basis of $R^{2 N}$ as claimed. The norm of a basis vector is $2^{N / 2}$.

The matrix

$$
M=\left[z_{1}, z_{2}, \cdots, z_{2} N\right]
$$

is orthogonal, and

$$
M M^{T}=2^{N} I
$$

where $I$ is the $2^{N}$ by $2^{N}$ identity matrix. Also note that all elements of $\boldsymbol{M}$ are either 1 or -1 . Orthcgonal matrices whose elements are 1 or -1 are called Hadamard matrices, and have been used in other fields of signal processing, like image encoding [19].

If the ordering of the basis vectors is chosen as the one which results from the same ordering of the bits $z_{0}, z_{1}, \cdots$, $z_{N-1}$ as in (A-3), $M$ is also symmetric and, from (A-6), is its own inverse. This ordering was used in writing (A-3).

These properties of $\boldsymbol{M}$ are useful in computing the coefficients of expansion (A-1) when it is written in terms of a binary variable which assumes values 1 and -1 . To compute the coefficients of (A-1) we must solve the system of $2^{N}$ linear equations

$$
M^{T} c=f
$$

which admits the closed form solution

$$
c=\frac{1}{2^{N}} M f
$$

Expression (A-8) allows the direct calculation of the coefficients of the expansion, once the values of $f$ for all possible sequences of $N$ bits are known. It is at the same time another proof of the validity of expansion (A-1) since $M$, being orthogonal, is always nonsingular, and so system (A-7) can always be solved.

\section{APPENDIX B}

If $u_{n}(r)$ given by (13) is placed at the input of $R$ interleaved transversal filters with unit sample responses $\nu_{r}(n)$, each sampling at the data rate, the output of the $r$ th filter will be

$$
y_{n}(r)=\sum_{k} \nu_{r}(k) u_{n-k}(r)=\sum_{k} \nu_{r}(k) x_{n-k} T \cdot g(r)
$$

and the power of the output signal will be

$$
\begin{aligned}
E\left\{y_{n}^{2}(r)\right\}= & g^{T}(r) \cdot\left[\sum _ { k _ { 1 } , k _ { 2 } } \nu _ { r } ( k _ { 1 } ) \nu _ { r } ( k _ { 2 } ) E \left\{x_{n-k_{1}}\right.\right. \\
& \left.\left.\cdot x_{n-k_{2}}{ }^{T}\right\}\right] \cdot g(r) \\
= & g^{T}(r)\left[\sum_{k} \sum_{m=-\infty}^{\infty} v_{r}(k) \nu_{r}(k+m) A(m)\right] g(r)
\end{aligned}
$$

where

$$
A(m)=E\left[x_{n} x_{n+m}^{T}\right]
$$

is a $2^{N} \times 2^{N}$ matrix. Because component 0 of vector $x_{n}$ is always 1 , element $A_{00}(m)$ is 1 for all $m$. The other elements of $\boldsymbol{A}(m)$ are either 0 or 1 and all vanish for $|m| \geqslant N$. Thus, we can express

$$
\boldsymbol{A}(m)=\boldsymbol{B}+\boldsymbol{C}(m)
$$

where

$$
\boldsymbol{B}=\left[\begin{array}{ccccc}
1 & 0 & 0 & \cdots & 0 \\
0 & 0 & 0 & \cdots & 0 \\
0 & 0 & 0 & \cdots & 0 \\
\cdot & \cdot & . & \cdots & . \\
0 & 0 & 0 & \cdots & 0
\end{array}\right]
$$

and $C(m)$ is identically 0 for $|m| \geqslant N$ but is different from 0 for $|m|<N$. It is interesting to note also that $C(m)$ depends only on $N$, and so a universal table of $C(m)$ matrices could be computed as a function of $N$.

In the special case when the basis vectors defined in Appendix $\mathrm{A}$ are taken in the order of (A-3), matrix $C(m)$ has the form

$$
\begin{aligned}
& C_{i, j}(m)=C_{j, i}(m)=\delta_{j, 2} m_{i} \\
&\left(1 \leqslant i \leqslant 2^{N-m}-1,1 \leqslant j \leqslant 2^{N}-1\right)
\end{aligned}
$$

$C_{0,0}(m)=0$.

Now

$$
\begin{aligned}
E\left\{y_{n}{ }^{2}(r)\right\}= & \alpha_{0}(r)\left|g_{0}(r)\right|^{2}+g^{T}(r) \\
& \cdot\left[\sum_{m=-N+1}^{N-1} \beta_{m}(r) C(m)\right] g(r)
\end{aligned}
$$

where

$$
\begin{aligned}
& \beta_{m}(r)=\sum_{k=-\infty}^{\infty} \nu_{r}(k) \nu_{r}(k+m) \\
& \alpha_{0}(r)=\sum_{m=-\infty}^{\infty} \beta_{m}(r)=\left(\sum_{k=-\infty}^{\infty} \nu_{r}(k)\right)^{2} .
\end{aligned}
$$

The first term in (B-8) represents the power of the timing tone at the output of the $r$ th filter, whereas the second term represents the jitter power, or the variance of the timing signal.

If we define

$$
M=\sum_{m=-N+1}^{N-1} \beta_{m}(r) C(m)
$$

then (15) results.

For the special case of WDM with an averaging filter of length $K$ as in (19) or (20), we have

$$
\beta_{m}=\left\{\begin{array}{cc}
\frac{(K-|m|)}{K^{2}} & (|m|<K) \\
0 & (|m| \geqslant K)
\end{array}\right.
$$

$\alpha_{0}=1$ 
If $K \gg N, M$ can be approximated as

$$
M=\left(\frac{1}{K}\right) \sum_{m=-N+1}^{N-1} \mathrm{C}(m) .
$$

For a resonator centered at $\omega=2 \pi$,

$$
\begin{aligned}
& \nu(n)=e^{-\alpha n} \\
& \beta_{m}=\frac{e^{-\alpha|m|}}{1-e^{-2 \alpha}}
\end{aligned}
$$

and

$$
\alpha_{0} \doteq \frac{1}{\left(1-e^{-\alpha}\right)^{2}}
$$

If we assume, as is usually the case, that the resonator has very small bandwidth, then $\alpha \ll 1$, and $M$ can be approximated as

$$
M=\frac{1}{1-e^{-2 \alpha}} \sum_{m=-N+1}^{N-1} C(m)
$$

\section{ACKNOWLEDGMENT}

We would like to acknowledge Dr. J. L. Massey for bringing to our attention some earlier literature [2] where expansion (A-1) is presented under the name of "algebraic normal form for Boolean functions," in the context of switching circuit design and error correction.

\section{REFERENCES}

[1] O. Agazzi, D. G. Messerschmitt, and D. A. Hodges, "Nonlinear echo cancellation of data signals," IEEE Trans. Commun., vol. COM-30, pp. 2421-2433, Nov. 1982.

[2] D. E. Muller, "Application of Boolean algebra to switching circuit design and to error detection," IRE Trans. Electron. Comput., vol. EC-3, pp. 6-12, Sept. 1954.

[3] T. Suzuki, H. Takatori, M. Ogawa, and K. Tomooka, "Line equalizer for a digital subscriber loop employing switched capacitor technology," IEEE Trans. Commun., vol. COM-30, pp. 2074-2082, Sept. 1982.

[4] L. E. Franks, "Carrier and bit synchroniżation in data communication-A tutorial review," IEEE Trans. Commun., vol. COM-28, pp. 1107-1121, Aug. 1980.

[5] _ _ "Synchronization subsystems: Analysis and design," in Digital Communications-Satellite/Earth Station Engineering, K. Feher; Ed. Englewood Cliffs, NJ: Prentice-Hall, 1983, ch. 7.

[6] F. M. Gardner, Phaselock Techniques. New York: Wiley, 1979.

[7] K. H. Mueller and M. Muller, "Timing recovery in digital synchronous data receivers," IEEE Trans Commun., vol. COM-24, pp. 516531, May 1976.

[8] W. R. Bennett, "Statistics of regenerative data transmission," Bell Syst. Tech. J., vol. 37, pp. 1501-1542, Nov. 1958.

[9] B. R. Saltzberg, "Timing recovery for synchronous binary data transmission," Bell Syst. Tech. J., vol. 46, pp. 593 622, Mar. 1967.

[10] R. D. Gitlin and J. Salz, "Timing recovery in PAM systems," Bell Syst. Tech. J., vol. 46, pp. 593-622, Mar. 1967.

[11] L. E. Franks and J. P. Bubrouski, "Statistical properties of timing jitter in a PAM timing recovery scheme," IEEE Trans. Commun., vol. COM-22, pp. 913-920, July 1974.

[12] J. E. Mazo, "Jitter comparison of tones generated by squaring and fourth-power circuits," Bell Syst. Tech. J., vol. 57, pp. 1489-1498, May-Junie 1978.

[13] D. L. Duttweiler, "The jitter performance of phase-locked loops extracting timing from baseband data waveforms," Bell Syst. Tech. J., vol. 55, pp. 37-58, Jan. 1976.

[14] D. D. Falconer and J. Salz, "Optimal reception of digital data over the
Gaussian channel with unknown delay and jitter," IEEE Trans. Inform. Theory, vol. IT-23, pp. 117-126, Jan. 1977 .

[15] R. D. Gitlin and J. F. Hayes, "Timing recovery and scrambleers in data transmission," Bell Syst. Tech. J., vol. 54, pp. 569-593, Mar: 1975.

[16]. D. G. Messerschmitt, "Frequency detectors for PLL acquisition in timing and carrier recovery," IEEE Trans. Commun., vol. COM-27, pp. 1288-1295, Sept. 1979.

[17] O. Agazzi and A. Adan, "System level computer simulations of a hybrid digital subscriber loop," CENICE (Nat. Cen. Electron. Component Res.), Buenos Aires, Argentina, internal pub., June 1982.

[18] S. E. Nader and L. F. Lind, "Optimal datâ transmission filters," IEEE Trans. Circuits Syst., vol. CAS-26, pp. 36-45, Jan. 1979.

[19] W. K. Pratt, J. Kane, and H. C. Andrews, "Hadamard transform image coding," Proc. IEEE, vol. 57, Jan. 1969.

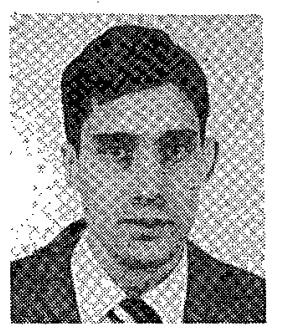

Oscar Agazzi (S'79-M'82) received the B.S. degree in electrical engineering and the Licentiate in physics from the National University of Cordoba, Cordoba, Argentina, in 1974 and 1975, respectively, and received the Ph.D. degree in electrical engineering from the University of California, Berkeley, in 1982

From 1974 to 1976 , he worked at the Nuclear Quadrupolar Resonance Research Group in the Department of Physics, National University of Cordoba, in charge of the design of instrumentation for physics research. During 1977 he worked as an independent consultant to private industry. From January to September 1978 he was with the Microelectronics Research Group at the Research Center of the Armed Forces, Buenos Aires, Argentina. He is with SENID (the Research and Development Service of the Argentine Navy) and CENICE (the Argentine National Center for Electronic Component Research), Buenos Aires.

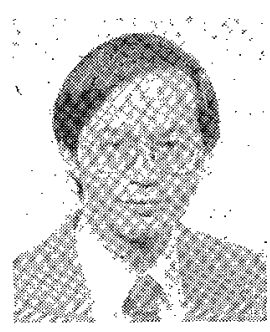

Chin-Pyng Jeremy Tzeng was born in Tainan, Taiwan, in July 1953. He received the B.S. degree from National Taiwan University in 1975, and the M.S. degree from the University of California at Berkeley in 1979, both in electrical engineering.

In 1979, he joined Rockwell International, Newport Beach, CA, where he was engaged in integrated circuit design. Since 1982, he has been with the University of California at Berkeley, working toward the Ph.D: degree with emphasis in integrated circuits and communications. He has several patents issued or pending.

Mr. Tzeng is a member of Eta Kappa Nu.

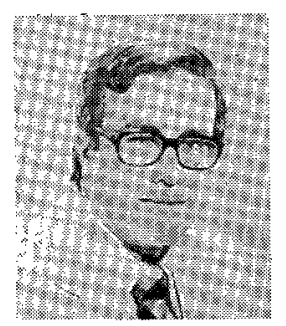

David G. Messerschmitt (S'65-M'68-SM'78$F^{\prime} 83$ ) received the B.S. degree from the University of Colorado, Boulder, in 1967, and the M.S. and Ph.D. degrees from the University of Michigan, Ann Arbor, in 1968 and 1971, respectively.

$\mathrm{He}$ is a Professor of Electrical Engineering and Computer Sciences at the University of California at Berkeley. From 1968 to 1977 he was a Member of the Technical Staff and later Supervisor at Bell Laboratories, Holmdel, NJ, where he did systems engineering, development, and research on digital transmission and digital signal processing (particularly relating to speech processing). His current research interests include analog and digital signal processing, speech processing, digital transmission, multiprocessor approaches to signal processing, digital subscriber loop transmission, adaptive filtering, communication network design and protocols, and computer-aided design of communications and signal processing systems. He has published over 60 papers and has 10 patents issued or pending in these fields. Since 1977 he has also served as a consultant to a number of companies, and is currently on the Board of Directors of Teknekron Infoswitch, Dallas, TX, and Scientific Director of Teknekron Communications Systemis Division; Berkeley. He has also organized and participated in a number of short courses and seminars devoted to continuing engineering education.

Dr. Messerschmitt is a member of Eta Kappa Nu, Tau Beta Pi, and Sigma $\mathrm{Xi}$, and has several best paper awards. He is currently a member of the Communication Theory Committee and the Board of Governors of the IEEE Communications Society, and has served as Editor for Transmission Systems of the IEEE TRANSACTIONS ON COMMUNICATIONS. 


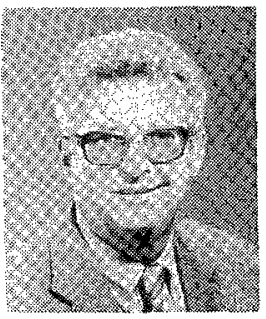

David A. Hodges (S'59-M'65-SM'71-F'77) received the B.E.E. degree from Cornell University, Ithaca, NY, and the M.S. and Ph.D. degrees in electrical engineering from the University of California, Berkeley.

From 1966 to 1970 he worked at Bell Laboratories, first in the components area at Murray Hill, $\mathrm{NJ}$, then as Head of the System Elements Research Department at Holmdel, NJ. Now he is Professor of Electrical Engineering and Computer Sciences at U.C. Berkeley, where he has been a member of the faculty since 1970 . He is coauthor or author of more than 80 published papers and is recorded as an inventor on five U.S. patents. Most of his publications relate to the design and application of digital and analog integrated circuits and VLSI. Currently he is involved with colleagues and students in research on analog and digital integrated circuit design, VLSI for telecommunications applications, and manufacturing information systems. In recent years he has served as a consultant to a variety of industrial firms including Intel Corporation, Seeq Technology, TRW, Xerox PARC, and Cyclotomics.

Prof. Hodges is a member of the National Academy of Engineering. He and two faculty colleagues, Paul R. Gray and Robert W. Broadersen, were corecipients of the 1983 IEEE Morris Liebmann Award for pioneering work on switched-capacitor circuits. 